K. MUTZE

DIE AKKOMMODATION DES MENSCHLICHEN AUGES 



\title{
DIE AKKOMMODATION DES MENSCHLICHEN AUGES
}

\author{
DIPL.-ING. K. M ÜTZ E \\ INSTITUT FÜR OPTIK UND FEINMECHANIK \\ DER DEUTSCHEN AKADEMIE DER WISSENSCHAFTEN ZU BERLIN \\ BERLIN-ADLERSHOF
}

MIT 113 ABBILDUNGEN UND 15 TABELLEN

1956

A K A D E M I E - V E R L A G - B E R L I N 
Erschienen im Akademie-Verlag GmbH., Berlin W 8, Mohrenstraße 39 Lizenz-Nr. $202-100 / 278 / 56$

Satz, Druck und Bindung: IV/2/14 - VEB Werkdruck Gräfenhainichen - 572 Schutzumschlag: Willi Bellert

Bestell- und Verlags-Nr. 5235

Printed in Germany 